\title{
Using PG-Liposome-Based System to Enhance Puerarin Liver-Targeted Therapy for Alcohol-Induced Liver Disease
}

\author{
Ying-Zheng Zhao, ${ }^{1,2}$ Lu Zhang, ${ }^{3}$ Pardeep K. Gupta, ${ }^{4}$ Fu-Rong Tian, ${ }^{1}$ Kai-Li Mao, ${ }^{1}$ Kai-Yan Qiu, ${ }^{1}$ Wei Yang, \\ Chuan-Zhu Lv, ${ }^{5}$ and Cui-Tao Lu ${ }^{1,2,6}$
}

Received 14 August 2015; accepted 30 September 2015; published online 11 January 2016

\begin{abstract}
A critical issue for alcohol-induced liver disease (ALD) therapeutics is the lack of a highly efficient delivery system. In this study, a Puerarin-propylene glycol-liposome system was prepared for the purpose of targeting puerarin, an isoflavon, to the liver. Transmission electron microscope (TEM) results showed the liposomes to be spherical in shape with an average diameter of $182 \mathrm{~nm}$ with a polydispersity index of 0.239 . The zeta potential of the particles was about $-30 \mathrm{mV}$. The entrapment efficiency of puerarin was above $90 \%$. MTT-based assay in HpeG2 cells showed no significant cytotoxicity in the presence of up to $25 \%$ concentration of the system containing $3 \%$ puerarin. In vivo performance of this system was studied in mice. Pharmacokinetics and distribution of puerarin-PG-liposome system was studied relative to puerarin solution at the same dose levels. The results show that puerarin-PGliposome prolonged drug retention time and decreased elimination of puerarin in mice (AUC of liposome system and solution was 9.5 and $4.0 \mathrm{mg} \mathrm{h} \mathrm{L}^{-1}$, respectively). Furthermore, propylene glycol (PG)-liposome system enhanced puerarin distribution into liver and spleen, while decreasing puerarin distribution in other tissues. Overall, the puerarin-PG-liposome system showed enhanced therapeutic effect in mice with ALD.
\end{abstract}

KEY WORDS: alcohol-induced liver disease; distribution; pharmacokinetics; propylene glycol liposome; puerarin.

\section{INTRODUCTION}

Alcohol-induced liver disease (ALD) progresses from early steatosis, inflammation, necrosis, to fibrosis/cirrhosis and/or hepato-cellular carcinoma (1). Despite significant advances in understanding of the pathogenesis of alcohol-related liver injury, there are no FDA-approved treatments for ALD (2-4). At present, abstinence remains the cornerstone for successful treatment of ALD (5).

Based on thousands of years of clinical experience, some herbal medicines are potential sources of novel anti-hepatitis drugs that may be used in liver disease therapy (5-7). Puerarin is the main component of wild Gregory, which is used to treat various inflammatory diseases, including hepatitis (8-11). A recent study showed that puerarin has a protective effect on

\footnotetext{
${ }^{1}$ College of Pharmaceutical Sciences, Wenzhou Medical University, Wenzhou, Zhejiang 325035, China.

${ }^{2}$ The Second Affiliated Hospital of Wenzhou Medical University, Wenzhou, Zhejiang 325000, China.

${ }^{3}$ The Third People's Hospital of Hangzhou, Hangzhou, Zhejiang 310000, China.

${ }^{4}$ Philadelphia College of Pharmacy, University of the Sciences in Philadelphia, Philadelphia, Pennsylvania 19104, USA.

${ }^{5}$ Hainan Medical College, Haikou, Hainan 570102, China.

${ }^{6}$ To whom correspondence should be addressed. (e-mail: lctuua@yahoo.com.cn)
}

ALD (12). However, pharmacokinetic researches revealed that the biological half-life of puerarin was $0.65 \pm 0.20 \mathrm{~h}$ and $\mathrm{MRT}_{0-\infty}$ was $0.314 \pm 0.040 \mathrm{~h}$ in rats after $5 \mathrm{mg} \times \mathrm{kg}^{-1}$ puerarin single intravenous injection, and it was rapidly eliminated by the kidney (13-15). These pharmacokinetic features reduce the clinical application of puerarin in ALD.

Structurally, puerarin is an isoflavon and contains a glucoside residue in the basic structure of daidzein (Fig. 1). It is classified as a 5-HT2c and GABA receptor antagonist (16). Its hydrophilic nature allows the kidney to filter it unchanged, resulting in a relatively short half-life.

In order to improve the pharmacokinetic parameters of puerarin including its half-life, plasma clearance (CL), and bioavailability, suitable drug delivery systems are needed. Among various delivery systems, liposomes are the most favorable system because of their high biocompatibility and safety. Liposome consist small, spherical, and enclosed compartment separating an aqueous medium from another by phospholipid bilayer (17). Several classes of drugs, including anticancer and antimicrobial agents, chelating agents, peptide hormones, enzymes, proteins, vaccines, and genetic materials, have been incorporated into the aqueous or lipid phase of liposome, with various sizes, compositions, and other characteristics, to provide selective delivery to the target site for in vivo application (18). As drug carriers, liposome has many potential advantages, which include reducing drug toxicity, increasing drug stability, and enhancing drug concentration 


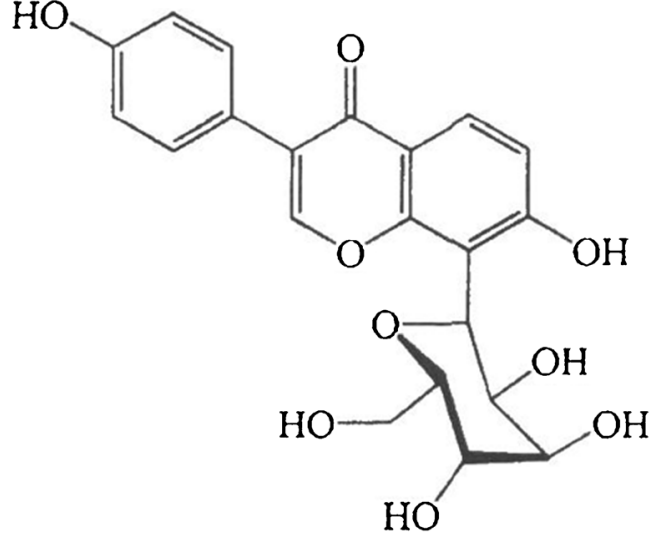

Fig. 1. Chemical structure of puerarin

in some organs, such as the liver or spleen (19-21). Since the first liposomal pharmaceutical product, Doxil, received FDA approval in 1995, liposomes have been widely applied as drug carriers. Modifications of liposomes include PEGylation, temperature-sensitive additives, cationic liposomes, and ethanol liposomes. Ethanol liposomes (ethosomes) typically contain $30-45 \%$ ethanol, which can provide the vesicles with soft flexible characteristics and allow easier penetration into cell lipid membrane (22). However, high concentration of ethanol in ethosomes solution is not stable. With ethanol evaporation during storage, ethosomes begin to gather, particle size increases, and loaded drug leaks from lipid vesicles. These problems will offset the advantages of ethosomes as the suitable delivery system for puerarin (23).

Propylene glycol (PG) has slightly higher oil-water partition coefficient than ethanol. In our previous experiments, a small amount of propylene glycol in the liposomal component has shown similar penetration ability as ethanol. Therefore, a new type of liposome-propylene glycol liposome (PGliposome) was developed (24). Based on our observation, PG-liposome can maintain stability during long-term storage.

In this paper, PG-liposome system was prepared as puerarin liver-targeted vector. The morphology of puerarinPG-liposome was observed by transmission electron microscope (TEM). The cellular uptake and cytotoxicity of puerarin-loaded PG-liposome (puerarin-PG-liposome) were observed and compared with puerarin solution. The pharmacokinetics and distribution of puerarin-PG-liposome were studied via intravenous administration. Routine blood indices were used to evaluate the therapeutic effect of puerarin-PGliposome on ALD mice.

\section{MATERIALS AND METHODS}

\section{Preparation of Puerarin-PG-Liposome and Puerarin-PG- Solution}

Preparation of Puerarin-PG-Liposome. Hydrogenated phosphatidylcholine $40 \mathrm{mg}$ (HPC; Doosan Corporation Biotech BU, Kyonggi Do, Korea), cholesterol 20 mg (Chol; Beijing Chemical Reagent Corporation, Beijing, China), tween802 mg (Hubei Biological Technology Company, Hubei Province, China), and puerarin $36 \mathrm{mg}$ (Mol wt.; 416, Tian fang Limit Company, Ningbo, China) were added into $2 \mathrm{~mL}$ propylene glycol. Propylene glycol solution was added slowly into $10 \mathrm{ml} \mathrm{5 \%}$ trehalose solution with constant rotation of $750 \mathrm{r} / \mathrm{min}$. Puerarin-PG-liposomes were formed after $30 \mathrm{~min}$ of continuous stirring containing puerarin at a concentration of $3 \mathrm{mg} / \mathrm{mL}$ in puerarin-PG-liposome suspension.

Preparation of Puerarin-PG-Solution. Tween-80 $2 \mathrm{mg}$ and puerarin $36 \mathrm{mg}$ were dissolved into $12 \mathrm{~mL}$ propylene glycol-water solution (propylene glycol, $5 \%$ trehalose solution $=1: 5$ ). The concentration of puerarin in puerarin-PG-solution was $3 \mathrm{mg} / \mathrm{mL}$.

\section{Characterization of Puerarin-PG-Liposomes}

\section{Morphology of Puerarin-PG-Liposomes}

The morphology of puerarin-PG-liposome was observed by transmission electron microscope (TEM) using negative staining with $1 \%$ phosphotungstic acid. Size distribution and surface charge of puerarin-PG-liposome was examined by Raman Spectrometer (M67205, Kehuayi Technology Company, Beijing, China). The average particle size and surface charge of puerarin-PG-liposome were reported from at least three samples.

\section{Puerarin Entrapment Efficiency}

The puerarin-PG-liposomes were separated from the free drug using Sephadex G-50 column. The effluent fluid containing puerarin-PG-liposome was collected and lyophilized at $5 \times$ $10^{-4} \mathrm{~Pa}$ pressure for $20 \mathrm{~h}$. Puerarin-PG-liposome lyophilized products were dissolved in anhydrous alcohol. The content of puerarin was determined by HPLC (chromatographic column $=$ Kromasil C18 $(250 \times 4.6 \mathrm{~mm}, 5 \mu \mathrm{m})$; mobile phase; methanol = water (25:75); flow rate $=1.0 \mathrm{~mL} / \mathrm{min}$; UV detection wave length $=250 \mathrm{~nm})$. Each sample was analyzed in triplicate. Entrapment efficiency was calculated with equation as reported previously (25).

\section{Cytotoxicity In Vitro}

MTT-based assay was performed to evaluate the cytotoxic effects of puerarin-PG-liposome system, puerarin-PG-solution, and PG-liposome in HepG2 (gifted from the Institute of Biological \& Natural Medicine, Wenzhou Medical University) cells in vitro. Vector concentration was set at five levels-5, 10, 15,25 , and $50 \%$ concentration in final volume (Table I). HepG2 cells were propagated in Dulbecco's modified Eagle medium (DMEM) (Genome Inc., Zhejiang Province, China) containing $10 \%$ fetal calf serum, $100 \mathrm{IU} / \mathrm{mL}$ penicillin, $100 \mathrm{mg}$ / $\mathrm{mL}$ streptomycin, and $2 \mathrm{mmol} / \mathrm{L}$ L-glutamine at $37^{\circ} \mathrm{C}$ under a $5 \% \mathrm{CO}_{2}$ atm. Cells harvested in a logarithmic growth phase were seeded on 96-wells at a cell density of $0.5 \times 10^{4}$ cells per well. After incubating the cells in a logarithmic phase with various concentrations of puerarin-PG-liposome, puerarinPG-solution, or PG-liposome for $4 \mathrm{~h}$, MTT assay was performed and the percent cell viability was determined. 
Table I. Puerarin Concentration in Puerarin-PG-Solution, Puerarin-PG-Liposome, and PG-Liposome

\begin{tabular}{|c|c|c|c|c|c|c|}
\hline \multirow[t]{2}{*}{ Level } & \multicolumn{2}{|c|}{ Puerarin-PG-solution } & \multicolumn{2}{|c|}{ Puerarin-PG-liposome } & \multicolumn{2}{|l|}{ PG-liposome } \\
\hline & $\begin{array}{l}\text { Volume ratio } \\
\quad(\%)\end{array}$ & $\begin{array}{l}\text { Puerarin concentration } \\
\quad(\mu \mathrm{g} / \mathrm{mL})\end{array}$ & $\begin{array}{l}\text { Volume ratio } \\
\quad(\%)\end{array}$ & $\begin{array}{l}\text { Puerarin concentration } \\
(\mu \mathrm{g} / \mathrm{mL})\end{array}$ & $\begin{array}{l}\text { Volume ratio } \\
(\%)\end{array}$ & $\begin{array}{l}\text { Puerarin concentration } \\
\quad(\mu \mathrm{g} / \mathrm{mL})\end{array}$ \\
\hline 1 & 5 & 100 & 5 & 100 & 5 & 0 \\
\hline 2 & 10 & 200 & 10 & 200 & 10 & 0 \\
\hline 3 & 15 & 300 & 15 & 300 & 15 & 0 \\
\hline 4 & 25 & 500 & 25 & 500 & 25 & 0 \\
\hline 5 & 50 & 1000 & 50 & 1000 & 50 & 0 \\
\hline
\end{tabular}

\section{Cellular Uptake In Vitro}

Based on results from toxicity study, puerarin-PGliposome and puerarin-PG-solution concentration was maintained below $25 \%$ in the drug cellular uptake study. Drug permeation into HepG2 cells was performed in 24-well cell culture plates (Coster Inc, USA). Cell suspension volume of $300 \mu \mathrm{L}\left(8 \times 10^{5}\right.$ cells $) /$ well was seeded in each well. After incubating the cells for $24 \mathrm{~h}, 250 \mu \mathrm{L}$ puerarin-PG-liposome or puerarin-PG-solution (puerarin concentration: $2 \mu \mathrm{g} / \mu \mathrm{L}$ ) were added. The medium was removed at different time intervals $(0,30,60,90,120,180$, and $240 \mathrm{~min})$, and cells were washed four times with PBS at $4^{\circ} \mathrm{C}$. Cells were then suspended and digested by incubation with $500 \mu \mathrm{L} 0.1 \%$ Triton X-100 overnight at $4^{\circ} \mathrm{C}$. This mixture was filtered through $0.45 \mu \mathrm{m}$ filter, and the extent of puerarin uptake into the cells was determined by HPLC (chromatographic column $=$ Kromasil $\mathrm{C} 18(250 \times 4.6 \mathrm{~mm}, 5 \mu \mathrm{m})$; mobile phase; methanol $=$ water (25:75); flow rate $=1.0 \mathrm{~mL} / \mathrm{min}$; UV detection wave length $=$ $250 \mathrm{~nm})$.

\section{Pharmacokinetics and Distribution of Puerarin-PG-Liposome In Vivo}

Swiss hasse mice (purchased from the Laboratory Animal Center of Wenzhou Medical University) were fasted for at least $12 \mathrm{~h}$ prior to the pharmacokinetic experiments while given water ad libitum. Each animal (equal number of male mice, $n=6$ each group) received $5 \mathrm{mg} \mathrm{kg}^{-1}$ dose of puerarin by tail vein either in PG-liposome or PG-solution formulation. After injection, the mice were sacrificed at $0,0.083,0.167$, $0.333,0.667,1.333,2.0,4.0,8.0,12$, and $24 \mathrm{~h}$. Blood samples were collected in heparinized tubes, and related organs (including spleen, kidney, liver, lung, and heart) were also collected in tubes from all mice. After weighing, tissue samples were processed with methanol $(\mathrm{pH}=9)$ by tip sonication for $10 \mathrm{~min}$ in a $4^{\circ} \mathrm{C}$ water bath, followed by centrifugation at $15,000 \mathrm{rpm}$. The supernatant fluid was transferred into blank tubes. Puerarin concentration in plasma or tissues was determined by HPLC as described in "Puerarin Entrapment Efficiency" section.

Puerarin concentration profiles in plasma and tissues were analyzed with DAS (Drug Analysis System 1.0). Pharmacokinetics was investigated by evaluating area under the curve from time 0 to $12 \mathrm{~h}\left(\mathrm{AUC}_{12 \mathrm{~h}}\right)$, terminal half-life $\left(t_{1 / 2}\right)$, mean residence time (MRT), total body clearance $(\mathrm{CL})$, and volume of distribution at steady state (Vss).

\section{Preliminary Evaluation of the Therapeutic Effect of Puerarin- PG-Liposome on ALD Mice}

Animal experiments in this study were carried out in compliance with the guidelines for the animal experimental center of Wenzhou Medical College. Swiss hasse mice were pair-fed Lieber DeCarli liquid diet containing 5\% ethanol, or a control diet in which ethanol was substituted a

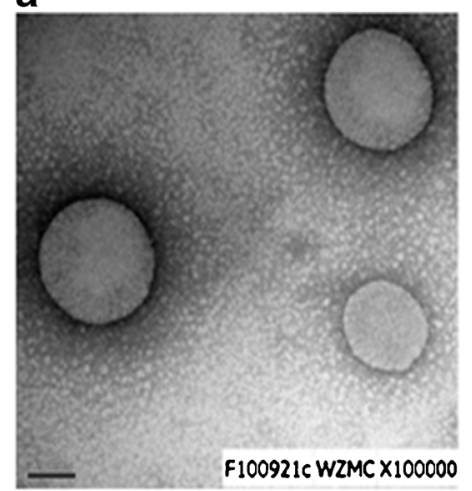

b

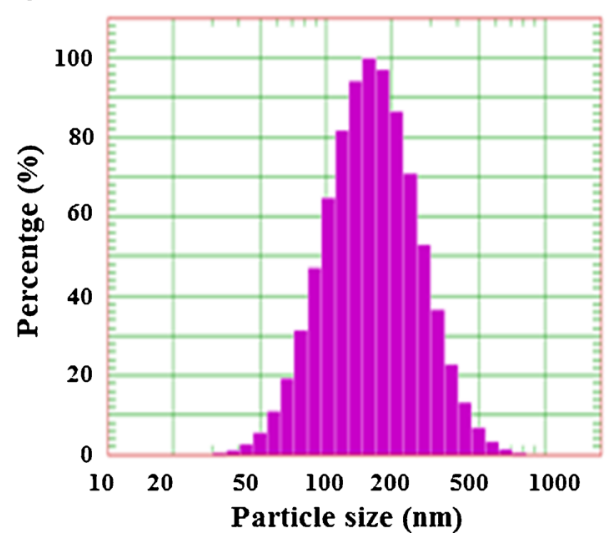

C

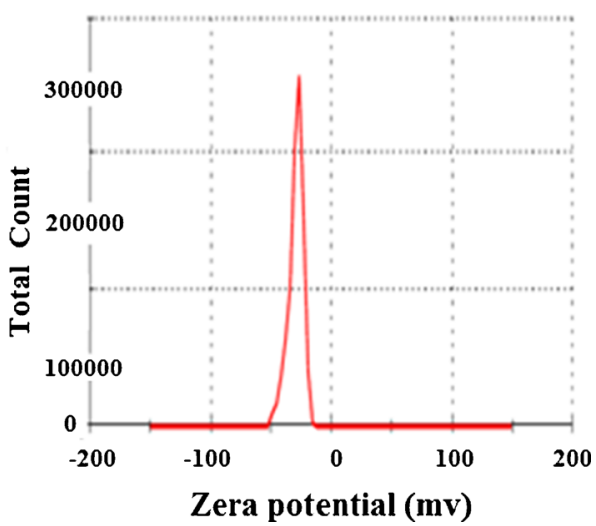

Fig. 2. Physical characteristics of puerarin-PG-liposome: a microphotographs of liposome $(\times 100,000)$, observed via transmission electron microscopy; b size distribution of liposome; $\mathbf{c}$ zeta potential of liposome 

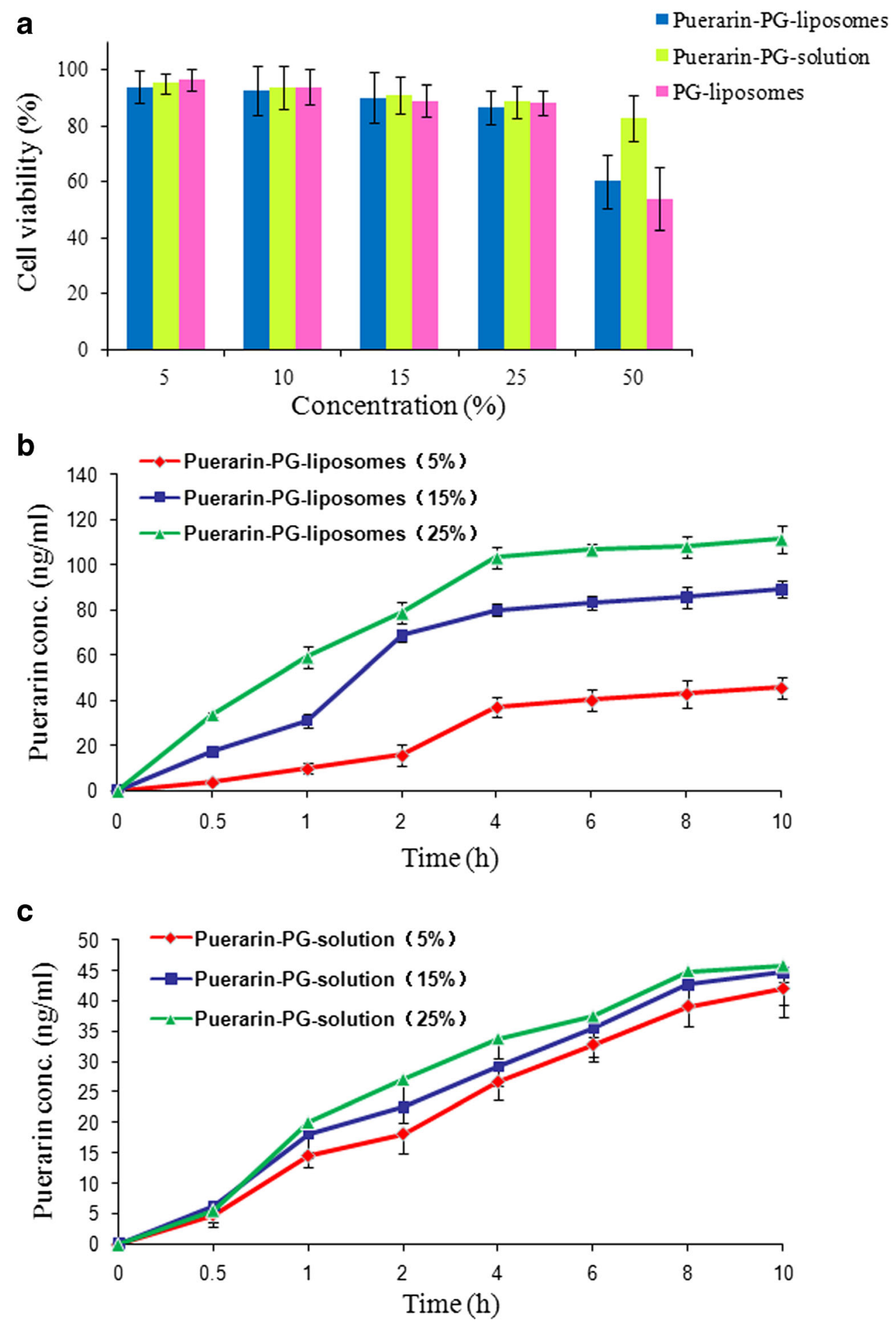

Fig. 3. Cytotoxicity and puerarin cellular uptake of different treatment: a viability of HepG2 cells after exposure to puerarin-solution, puerarin-PG-liposome, and PG-liposome respectively at different concentration levels; b cellular uptake of puerarin after puerarin-PG-liposome administration; c cellular uptake of puerarin after puerarin-solution administration. All values were shown as mean $\pm \mathrm{SD}, n=5$

iso-calorically by dextrin maltose for 8 weeks. Ethanol was introduced gradually by increasing the content by $1 \%$ every day until the mice were consuming diets containing $5 \%$ ethanol for up to 8 weeks. During the entire feeding period, the mice of model group and the mice treated with puerarin-PG-solution and puerarin-PG-liposome were observed to consume similar volumes of ethanol diets daily (calculated according to the ethanol quality: $8 \mathrm{~g}$ / 
Table II. Pharmacokinetic Parameters After Intravenous Administration of Puerarin-PG-Liposome or Puerarin-PG-Solution at a Dose of 5 $\mathrm{mg} / \mathrm{kg}$ in Mice

\begin{tabular}{lcc}
\hline Parameter & $\begin{array}{c}\text { Puerarin-PG- } \\
\text { solution }\end{array}$ & $\begin{array}{c}\text { Puerarin-PG- } \\
\text { liposome }\end{array}$ \\
\hline $\begin{array}{c}\mathrm{AUC}_{(0-8)} / \\
\mathrm{mg} \mathrm{h} \mathrm{L}^{-1}\end{array}$ & $4.02 \pm 0.537$ & $9.545 \pm 3.88^{*}$ \\
$\mathrm{t}_{1 / 2(\mathrm{ke})} / \mathrm{h}$ & $0.745 \pm 0.378$ & $3.105 \pm 1.737^{*}$ \\
$\mathrm{CL} / \mathrm{L} \mathrm{h}^{-1}$ & $4.847 \pm 0.681$ & $1.903 \pm 1.096^{*}$ \\
$\mathrm{MRT}_{(0-8)} / \mathrm{h}$ & $1.226 \pm 0.251$ & $2.111 \pm 0.612^{*}$ \\
$\mathrm{~V}_{\mathrm{d}} / \mathrm{L}$ & $5.134 \pm 2.445$ & $9.820 \pm 6.296$ \\
\hline
\end{tabular}

Values represent mean $\pm \mathrm{SD}(n=5)$. Asterisk represents $P<0.05$ vs. puerarin-PG-solution

(kg/day). Five groups of mice (seven each) were separated as follows:

1. Blank control group: pair-fed untreated mice

2. Model group: model alcohol feeding mice

3. Negative control group: model mice treated with PGliposome without puerarin $(40 \mathrm{~mL} / \mathrm{kg})$

4. Treatment group I: model mice treated with puerarinPG-solution (40 mL/kg)

5. Treatment group II: model mice treated with puerarinPG-liposome $(40 \mathrm{~mL} / \mathrm{kg})$

In the treatment groups, drugs were administered by tail vein every day for 8 weeks. The mice in normal and model control groups were given the same volume of normal saline.

Routine blood indices were used to evaluate the therapeutic effect of puerarin-PG-liposome on ALD mice. Serum alanine transaminase (ALT), aspartate aminotransferase (AST), triglycerides, and cholesterol were determined using a clinical chemistry analyzer system (PROCHEM-V; Drew Scientific, Barrow-in-Furness, UK).

\section{Statistical Analysis}

One-way ANOVA and Student's $t$ test or Kruskal-Wallis test were adopted for statistical comparison using the SAS
8.01 (1999-2000, SAS Institute Inc., Cary, NC, USA). The data difference was considered to be statistically significant when the $P$ value was less than 0.05 .

\section{RESULT AND DISCUSSION}

\section{Characterization of Puerarin-PG-Liposomes}

As reported above, micromorphology of liposome was observed via transmission electron microscopy. As shown in Fig. 2, puerarin-PG-liposome was characterized by spherical morphology, and no aggregation or fusion was observed. Puerarin-PG-liposome showed a mean particle size of $182 \mathrm{~nm}$, with a polydispersity index of 0.239 . The zeta potential of puerarin-PG-liposome was slightly negative (about $-29.4 \mathrm{mV}$ ). In addition, the entrapment efficiency of puerarin-PG-liposome reached $93.6 \pm 1.7 \%$, which was much higher than puerarin solid lipid nanoparticle and puerarinloaded liposome reported previously (26). The presence of propylene glycol in liposomal structure appears to increase the entrapment efficiency of puerarin-PG-liposome system when compared with the conventional puerarin-loaded liposomes.

\section{Results of Cytotoxicity Assay and Drug Cellular Uptake}

From MTT results, liposome concentration and drug concentration contributed to the cytotoxicity of puerarinPG-liposome and puerarin-PG-solution (both puerarin concentration in PG-liposome and PG-solution was $3 \mathrm{mg} / \mathrm{mL}$ ). As shown in Fig. 3a, puerarin-PG-liposome under low concentration $(\leq 25 \%)$ showed little difference in cell viability with puerarin-PG-solution $(P>0.05)$. At high concentration $(>50 \%$,$) , however, puerarin-PG-liposome caused$ almost half of the cells to die (cell viability $=60.2 \%$ ). In contrast, cell viability of puerarin-PG-solution was $82.9 \%$, which was much higher than that of puerarin-PG-liposome $(P<0.05)$. From these result, it was concluded that puerarin-PG-liposome at low concentration $(<25 \%)$ had good safety profile for HepG2 cells. In order to avoid any cytotoxic effects of PG-liposome, cellular uptake study

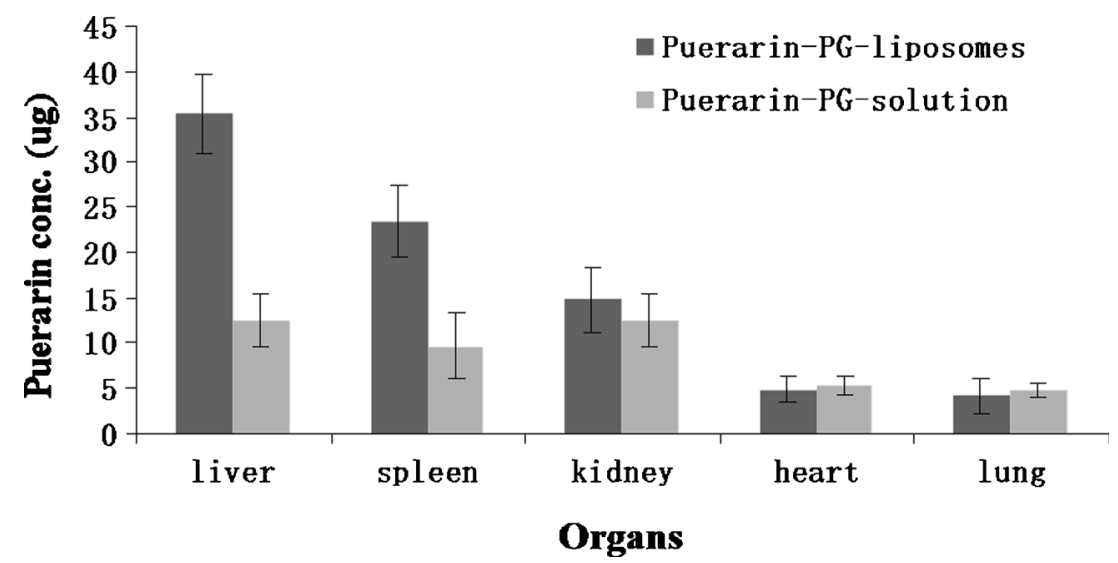

Fig. 4. Puerarin content in organs at $6 \mathrm{~h}$ after i.v. single dose of $5 \mathrm{mg} / \mathrm{kg}$ puerarin with puerarin-PG-liposome and puerarin-PG-solution. (All values were shown as mean $\pm \mathrm{SD}, n=5$ ) 
Table III. Plasma Biochemical Indexes of Mice After Different Treatment

\begin{tabular}{llccc}
\hline Group & ALT (U/L) & AST (U/L) & TCHOL (mmol/L) & TRIG (mmol/L) \\
\hline Normal group & $37.5 \pm 5.3$ & $74.5 \pm 8.8$ & $2.3 \pm 1.1$ & $0.8 \pm 0.5$ \\
Model group & $82.3 \pm 10.4$ & $170.0 \pm 7.9$ & $3.5 \pm 0.9$ & $2.1 \pm 0.8$ \\
Negative control group & $90.6 \pm 6.1$ & $172.8 \pm 3.3$ & $2.9 \pm 1.1$ & $2.3 \pm 0.6$ \\
Treatment group I & $65.8 \pm 6.7^{*}$ & $124.3 \pm 12.1^{*}$ & $3.2 \pm 1.2$ & $1.5 \pm 0.4$ \\
Treatment group II & $47.6 \pm 3.2^{*}$ & $80.5 \pm 5.0^{*}$ & $2.1 \pm 0.6$ & $1.3 \pm 0.6$ \\
\hline
\end{tabular}

Blank control group pair-fed untreated mice, Model group model alcohol feeding mice, Negative control group model mice treated with PGliposome without puerarin $(40 \mathrm{~mL} / \mathrm{kg})$, Treatment group I model mice treated with puerarin-PG-solution $(40 \mathrm{~mL} / \mathrm{kg})$, Treatment group II model mice treated with puerarin-PG-liposome $(40 \mathrm{~mL} / \mathrm{kg})$. Values represent mean $\pm \mathrm{SD}(n=7)$. Asterisk represents $P<0.05 v s$. Model group

was conducted with puerarin-PG-liposome concentration of less than $25 \%$.

Figure $3 b$, c shows the cellular uptake of puerarin-PGliposome or puerarin-PG-solution puerarin. Cellular uptake of puerarin-PG-liposome at each time point (2, 4, 6, 8, $12 \mathrm{~h}$ ) was significantly higher than that of puerarin-PG-solution $(P<0.05)$. With increasing liposome concentration, the rate and amount of drug uptake into cell was significantly increased. The sequence of the cell penetration efficiency for puerarin-PG-liposome was higher concentration (110 ng/ $\mathrm{mL}, 6 \mathrm{~h})>$ middle concentration $(88 \mathrm{ng} / \mathrm{mL}, 5 \mathrm{~h})>$ lower concentration $(60 \mathrm{ng} / \mathrm{mL}, 8 \mathrm{~h})>$ drug solution $(36 \mathrm{ng} / \mathrm{mL}$, $6 \mathrm{~h})$. But in puerarin-PG-solution, no dose-dependent relationship was observed. There are two ways for drug to enter cell membrane in PG-liposome: (1) free drug enters the cell by simple diffusion; (2) cell could take up drug loaded liposome particles by endocytosis (27). One of the previous studies showed that cell endocytosis was a dose-dependent process (28).

Propylene glycol (PG) has been widely utilized as a penetration enhancer and adjuvant in topical formulations. It has been already assayed as a component of lipid vesicles by Elsayed et al. who tested the PG-liposomes as carriers for skin delivery of cinchocaine (29). Therefore, PGliposomes have better penetration effects than PG-solution. With the endocytosis of liposomes, puerarin-PG-liposomes can realize high dose in cell. Therefore, the promoted cellular uptake of puerarin may be resulted from the enhanced membrane penetration of PG and the endocytosis of liposomal system.

\section{Pharmacokinetics and Distribution of Puerarin-PG-Liposome}

As shown in Table II, the main pharmacokinetic parameters of puerarin-PG-liposome were significantly different from those of puerarin-PG-solution. The AUC and $t_{1 / 2(\mathrm{ke})}$ of the puerarin-PG-liposome in plasma were 2.37 and 4.16 times of those of puerarin-PG-solution respectively $(P<0.05)$. Clearance $(\mathrm{CL})$ of puerarin-PG-liposome was much lower than that of puerarin-PG-solution (1.903 vs $4.847 \mathrm{~h}, P<0.05)$. From these results, it can be concluded that puerarin-PG-liposome prolonged drug retention time and decreased elimination of puerarin in vivo. Therefore, puerarin pharmacokinetic parameters in vivo can be improved with puerarin loaded in PG-liposome.

Figure 4 shows puerarin content in organs at $6 \mathrm{~h}$ after an i.v. single dose of $5 \mathrm{mg} / \mathrm{kg}$ puerarin with puerarin-PGliposome and puerarin-PG-solution. From the profile, it can be observed that puerarin-PG-liposome delivered a much higher amount of puerarin into liver and spleen $(P<0.05)$. Puerarin levels in liver and spleen of puerarin-PG-liposome were about twofold higher than that of puerarin-PG-solution. In kidney, however, puerarin amount of puerarin-PGliposome was slightly lower than that of puerarin-PGsolution $(P>0.05)$. In heart and lung, puerarin levels showed little difference between puerarin-PG-liposome and puerarinPG-solution $(P>0.05)$. Overall, this data shows that PGliposome enhanced puerarin distribution into liver and spleen at the expense of other organs. The distribution behavior of puerarin-PG-liposome would benefit the therapeutic effect of puerarin in liver and spleen diseases.

\section{Preliminary Evaluation of the Therapeutic Effect on ALD Mice}

Routine blood indices were used to evaluate of the therapeutic effect of puerarin-PG-liposome on ALD mice. As shown in Table III, there was a significant increase in serum ALT and AST activities after alcohol feeding $(P<0.05)$. Treatment with puerarin-PG-liposome (treatment group II) and puerarin-PG-solution (treatment group I) could significantly reduce both ALT and AST levels $(P<0.05)$. Furthermore, plasma levels of total cholesterol and triglyceride in puerarin-PG-liposome and puerarin-PG-solution groups were lower than those in the model group $(P>0.05)$. As expected, puerarin-PG-liposome (treatment group II) showed better curative effects than puerarin-PG-solution (treatment group I) on ALD mice according to all the plasma biochemical indexes.

\section{CONCLUSIONS}

The clinical utility of most conventional therapeutics is limited either by the inability to deliver therapeutic drug concentrations to the target tissues or by severe and harmful toxic effects on normal organs and tissues. In this paper, PGliposome was used to improve the pharmacokinetics and distribution of puerarin in vivo, which would enhance the therapeutic effect of ALD.

The results show that puerarin-PG-liposome had a homogeneous spherical shape, with an average diameter of $182 \mathrm{~nm}$ and a relatively narrow, monodisperse size distribution. From MTT assay, liposomal concentration less than $25 \%$ in final volume is a safe dosage range that can avoid severe cytotoxic effects. Pharmacokinetic data indicated that puerarin-PG-liposome increased the AUC in plasma, 
prolonged puerarin retention time, and decreased elimination of puerarin in vivo. Furthermore, PG-liposome enhanced puerarin distribution into liver and spleen, while decreasing puerarin distribution in other tissues. Preliminary observation of the therapeutic effect on ALD mice also showed the enhanced therapeutic effect of puerarin-PGliposome on ALD mice.

Although encouraging results have been observed in this study, puerarin-PG-liposome system's ability to provide promising therapeutic effects in patient population remains to be studied. For future development of PG-liposome system, the clinical therapeutic effects and toxic side effects of puerarinPG-liposome should be investigated. Therapeutic mechanism of puerarin-PG-liposome for ALD at a biological level should also be studied. With the further research, puerarin-PGliposome has a potential to be developed as a targeted delivery system for ALD.

\section{ACKNOWLEDGMENTS}

This research was supported by the National Natural Science Foundation of China (Grant Nos. 81360195, $81301982,81571392,81272160,81302726$, and 81460299), Zhejiang Provincial Foundation for Health Department (Grant No. 2015ZDA023), Medicine Grant from Wenzhou Bureau of Science and Technology (Grant No. Y2014730), Major Scientific Project of Guangdong Province (Grant No. 2012A080201010), and Science and Technology Program of Guangzhou, China (201508020001).

\section{Compliance with Ethical Standards}

Conflict of Interest. The authors report no conflicts of interest. The authors alone are responsible for the content and writing of this paper.

\section{REFERENCES}

1. Albano E. Alcohol, oxidative stress and free radical damage. Proc Nutr Soc. 2006;65:278-90.

2. Arteel GE. Alcohol-induced oxidative stress in the liver: in vivo measurements. Methods Mol Biol. 2008;447:185-97.

3. Vidali M, Stewart SF, Albano E. Interplay between oxidative stress and immunity in the progression of alcohol-mediated liver injury. Trends Mol Med. 2008;14:63-71.

4. Frazier TH, Stocker AM, Kershner NA, Marsano LS, McClain CJ. Treatment of alcoholic liver disease. Therap Adv Gastroenterol. 2011;4:63-81.

5. Hu S, Shen G, Zhao W, Wang F, Jiang X, Huang D. Paeonol, the main active principles of Paeoniamoutan, ameliorates alcoholic steatohepatitis in mice. J Ethnopharmacol. 2010;2(128):100-6.

6. Li FQ, Su H, Wang J, Liu JY, Zhu QG, Fei YB, et al. Preparation and characterization of sodium ferulate entrapped bovine serum albumin nanoparticles for liver targeting. Int J Pharm. 2008;349:274-82.

7. Cho K, Kim SJ, Park SH, Kim S, Park T. Protective effect of Codonopsislanceolata root extract against alcoholic fatty liver in the rat. J Med Food. 2009;12:1293-301.

8. Gao FY. Research progress in pharmacological actions of puerarin. Chin Tradit Herbal Drugs. 2003;34:7-8.

9. Singh AK, Jiang Y, Benlhabib E, Gupta S. Herbal mixtures consisting of puerarin and either polyenylphosphatidylcholine or curcumin provide comprehensive protection against alcohol- related disorders in $\mathrm{P}$ rats receiving free choice water and $15 \%$ ethanol in pure water. J Med Food. 2007;10:526-42.

10. Xie W, Du L. Diabetes is an inflammatory disease: evidence from traditional Chinese medicines. Diabetes Obes Metab. 2011;13:289-301.

11. Zheng P, Ji G, Ma Z, Liu T, Xin L, Wu H, et al. T Therapeutic effect of puerarin on non-alcoholic rat fatty liver by improving leptin signal transduction through JAK2/STAT3 pathways. Am J Chin Med. 2009;37:69-83.

12. Qu ZW, Wen CY, Wang AP, Ju WB, Ren AH, Liu MY, et al. Protective effect of puerarin on rats with alcoholic hepatitis. World Chin J Digestol. 2009;17:657-61.

13. Yuan M, Chen MS, Liu SM, Huang BY, Liu XW, Zhu L. Determination of puerarin in rat plasma by rapid resolution liquid chromatography tandem mass spectrometry in positive ionization mode. J Chromatogr B Analyt Technol Biomed Life Sci. 2011;879:1497-501.

14. Li Y, Pan WS, Chen SL, Xu HX, Yang DJ, Chan AS. Pharmacokinetic, tissue distribution, and excretion of puerarin and puerarin-phospholipid complex in rats. Drug Dev Ind Pharm. 2006;32:413-22.

15. Penetar DM, Teter CJ, Ma Z, Tracy M, Lee DY, Lukas SE. Pharmacokinetic profile of the isoflavonepuerarin after acute and repeated administration of a novel kudzu extract to human volunteers. J Altern Complement Med. 2006;12:543-8.

16. Overstreet DH, Kralic JE, Morrow AL, Ma ZZ, Zhang YW, Lee DY. NPI-031G (puerarin) reduces anxiogenic effects of alcohol withdrawal or benzodiazepine inverse or 5-HT2C agonists. Pharmacol Biochem Behav. 2003;75:619-25.

17. Panwar P, Pandey B, Lakhera PC, Singh KP. Preparation, characterization, and in vitro release study of albendazole-encapsulated nanosize liposomes. Int J Nanomedicine. 2010;5:101-8.

18. Chang H-I, Yeh M-K. Clinical development of liposome-based drugs: formulation, characterization, and therapeutic efficacy. Int J Nanomedicine. 2012;7:49-60.

19. Martins S, Sarmento B, Ferreira DC, Souto EB. Lipid-based colloidal carriers for peptide and protein delivery-liposomes versus lipid nanoparticles. Int J Nanomedicine. 2007;2:595-607.

20. Zhao YZ, Lu CT, Li XK, Tang QQ, Tian XQ, Zhao YP, et al. Improving the cardio protective effect of aFGF in ischemic myocardium with ultrasound-mediated cavitation of heparin modified microbubbles: preliminary experiment. J Drug Target. 2012;20:623-31.

21. Smola M, Vandamme T, Sokolowski A. Nanocarriers as pulmonary drug delivery systems to treat and to diagnose respiratory and non respiratory diseases. Int $\mathrm{J}$ Nanomedicine. 2008;3:1-19.

22. Touitou E, Dayan N, Bergelson L, Godin B, Eliaz M. Ethosomes novel vesicular carriers for enhanced delivery: characterization and skin penetration properties. J Control Release. 2000;65:403-18.

23. Zhao YZ, Lu CT, Zhang Y, Xiao J, Zhao YP, Tian JL, et al. Selection of high efficient transdermal lipid vesicle for curcumin skin delivery. Int J Pharm. 2013;454:302-9.

24. Zhang L, Lu CT, Li WF, Cheng JG, Tian XQ, Zhao YZ, et al. Physical characterization and cellular uptake of propylene glycol liposomes in vitro. Drug Dev Ind Pharm. 2012;38:365-71.

25. Zhao YZ, Sun CZ, Lu CT, Dai DD, Lv HF, Wu Y, et al. Characterization and anti-tumor activity of chemical conjugation of doxorubicin in polymeric micelles (DOX-P) in vitro. Cancer Lett. 2011;311:187-94.

26. Wang HG, Zhai GX, Lü QZ, Ling PX. Preparation and characterization of chitosan coated puerarin liposome. Zhong Yao Cai. 2007;30:89-92.

27. Breunig M, Bauer S, Goefferie A. Polymers and nanoparticles: intelligent tools for intracellular targeting? Eur J Pharm Biopharm. 2008;6:112-28.

28. Torchilin VP. Recent approaches to intracellular delivery of drugs and DNA and organelle targeting. Annu Rev Biomed Eng. 2006;8:343-75.

29. Elsayed MM, Abdallah OY, Naggar VF, Khalafallah NM. Lipid vesicles for skin delivery of drugs: reviewing three decades of research. Int J Pharm. 2007;332(1-2):1-16. 